04

\title{
Тороидальные альфвеновские моды в плазме токамака Глобус-М
}

\author{
(C) В.К. Гусев ${ }^{1}$, А.А. Мартынов ${ }^{2}$, С.Ю. Медведев ${ }^{2}$, Ю.В. Петров ${ }^{1, \uparrow, ~}$ \\ М.И. Патров ${ }^{1}$, А.Ю. Тельнова ${ }^{1}$ \\ ${ }^{1}$ Физико-технический институт им. А.Ф. Иоффе РАН, Санкт-Петербург, \\ Россия \\ ${ }^{2}$ Институт прикладной математики им. М.В. Келдыша РАН, Москва, \\ Россия \\ ๑ E-mail: yu.petrov@mail.ioffe.ru
}

Поступило в Редакцию 11 сентября 2017 г.

Для выяснения условий возбуждения TAE-мод (toroidal Alfven eigenmode) и их влияния на удержание быстрых частиц в сферическом токамаке Глобус-М при помощи кода KINX проведены расчеты магнитогидродинамических спектров реконструированных диверторных равновесных конфигураций со значением фактора запаса устойчивости на магнитной оси $q_{0}>1$. Исследована чувствительность частот ТАЕ-мод с тороидальным волновым числом $n=1$ к типу граничных условий и выбору граничной поверхности. Найдено, что частоты мод с доминирующими полоидальными гармониками $m=1$ и 2 в щели континуума существенно выше, чем наблюдаемые в спектрограммах сигналов от катушеук Мирнова, особенно в предположении свободной границы плазмы и с учетом ее сжимаемости. ТАЕ-моды с более низкими частотами и более высокими полоидальными волновыми числами, локализованные вблизи границы плазмы, могут быть ответственны за колебания, наблюдаемые в эксперименте. Однако для этих мод характерно взаимодействие с континуумом и, возможно, связанное с этим затухание.

DOI: $10.21883 /$ PJTF.2018.02.45466.17034

В экспериментах с инжекцией пучка нейтральных атомов на ранней стадии разряда токамака Глобус-М наблюдались неустойчивости, возбуждаемые быстрыми ионами в диапазоне частот 50-200 kHz, которые были идентифицированы как тороидальные альфвеновские моды (ТАЕ) [1-3]. Цель настоящей работы - промоделировать структуру и частотный спектр альфвеновских мод и провести их сравнение с экспериментально наблюдаемыми. Для моде- 
лирования был выбран разряд с инжекцией водородного пучка в дейтериевую плазму (см. рис. 2, $d$ в работе [2], разряд 31497), в котором основная интенсивная мода развивается на частоте $\sim 100 \mathrm{kHz}$.

Малое аспектное отношение плазмы сферического токамака приводит к появлению широкой щели в континууме за счет взаимодействия мод с соседними номерами полоидальных гармоник $m$ и $m+1$. На рис. 1, a показана структура непрерывного альфвеновского спектра (показатель адиабаты $\Gamma=0$ ), рассчитанного по коду KINX [4] с использованием экспериментального профиля массовой плотности для квазинейтральной дейтериевой плазмы. В этой щели существует несколько глобальных ТАЕ-мод вблизи верхней и нижней границ континуума (сплошные горизонтальные линии на рис. 1,a). Заметим, что граничные условия на свободной границе плазмы (радиус проводящей стенки $1.3 a$, где $a$ - радиус границы плазмы) приводят к заметному увеличению частот глобальных ТАЕ-мод (рис. 1, $a$, штрихпунктирные горизонтальные линии). При этом выбор в качестве границы плазмы сепаратрисы магнитного поля или магнитной поверхности внутри сепаратрисы, определяемой заданной долей полоидального потока, не приводит к существенному изменению частот внешних ТАЕ-мод, что является следствием большого шира у границы плазмы сферического токамака. Помимо ТАЕ-мод с частотами, попадающими в основную тороидальную щель, расположенную вблизи магнитной поверхности с $q=1.5$, в идеальном магнитогидродинамическом спектре существуют глобальные моды с частотами, попадающими в непрерывный спектр. Частота и полоидальные числа этих мод ближе к экспериментально наблюдаемым. На рис. 2, $a, b$ показаны структуры таких мод с относительно большими номерами полоидальных гармоник, которые соответствуют тороидальному „перезамыканию“ ветвей континуума на магнитных поверхностях с $q=2.5$, 3.5. На всех рисунках линий уровня темный цвет соответствует максимумам смещения плазмы.

В более сложной модели, предполагающей сжимаемость плазмы (показатель адиабаты $\Gamma=5 / 3$ ), участвуют профили давления плазмы, полученные на основе данных об электронной температуре и заданного соотношения ионной и электронной температур $\left(T_{i} / T_{e}=0.5\right)$, что дает хорошее совпадение с профилями давления из восстановленного равновесия. Учет сжимаемости приводит к зацеплению альфве-

Письма в ЖТФ, 2018, том 44, вып. 2 


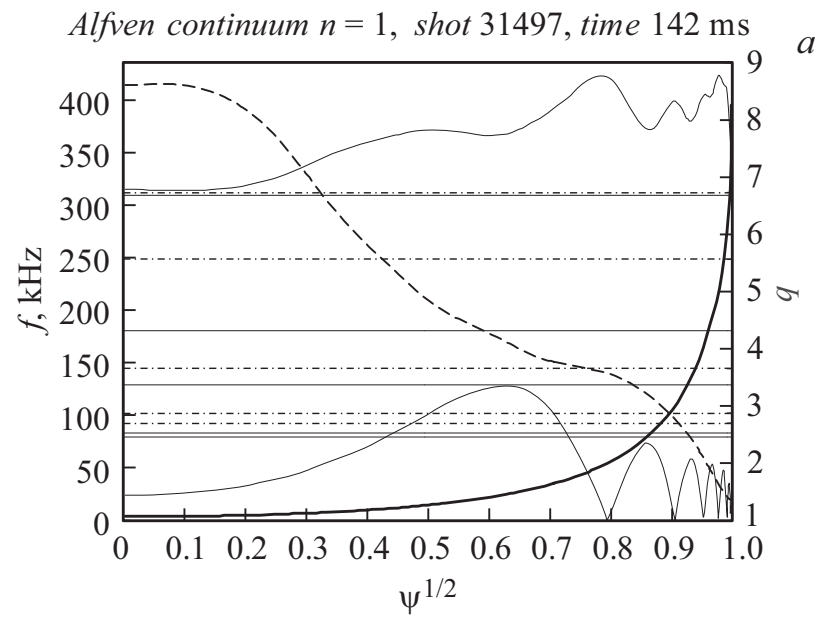

Alfen/sound continuum $n=1$, shot 31497 , time $142 \mathrm{~ms}$

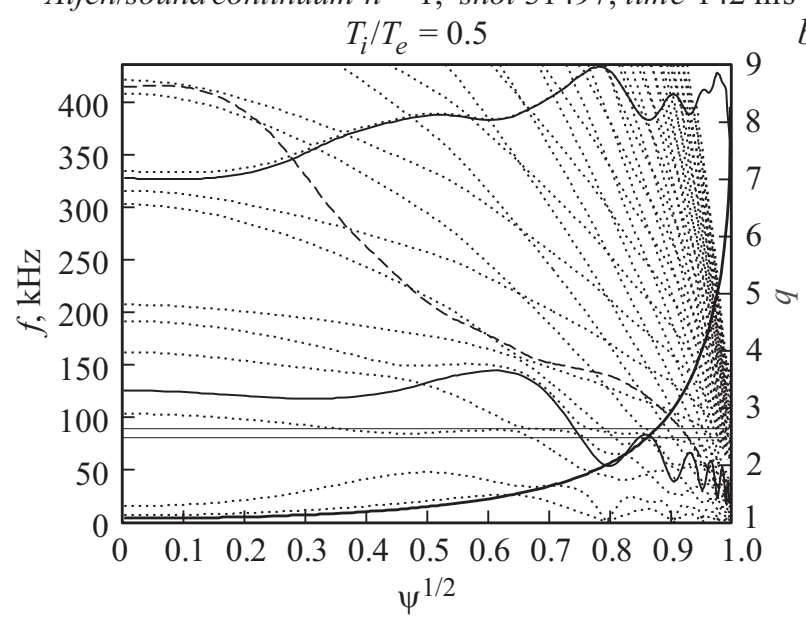

Рис. 1. Структура непрерывного альфвеновского спектра для тороидального волнового числа $n=1$ (разряд 31497, время $142 \mathrm{~ms}$ ). Профиль фактора запаса устойчивости $q$ показан жирной линией, экспериментальный профиль массовой плотности - штриховой линией. Горизонтальные линии соответствуют частотам глобальных мод. $a-$ альфвеновский спектр (показатель адиабаты $\Gamma=0$ ), $b-$ альфвеновский/звуковой спектр $(\Gamma=5 / 3)$ показан пунктирными линиями, спектр в модели медленного звука представлен сплошными линиями.

5* Письма в ЖТФ, 2018, том 44, вып. 2 

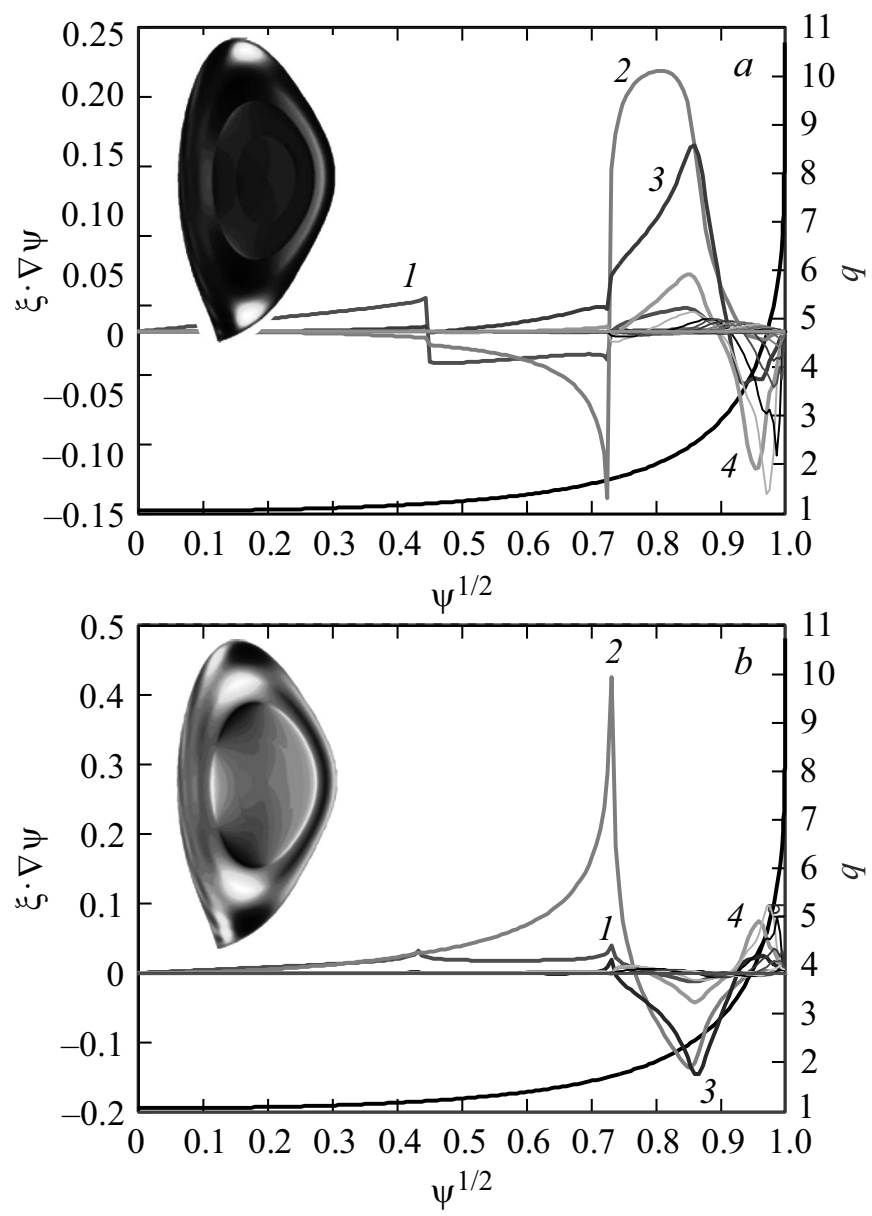

Pис. 2. Структура ТАЕ-мод в альфвеновском спектре $(\Gamma=0)$ с частотами $83(a)$ и $79 \mathrm{kHz}(b)$. Показаны гармоники смещения плазмы, нормального к магнитным поверхностям, $\xi \cdot \nabla \psi$ в координатах с выпрямленными силовыми линиями и линии уровня $\xi \cdot \nabla \psi|\nabla \psi|$ (темный цвет - максимум смещения). Цифры около кривых соответствуют номерам полоидальных гармоник $m=1,2,3,4$. 

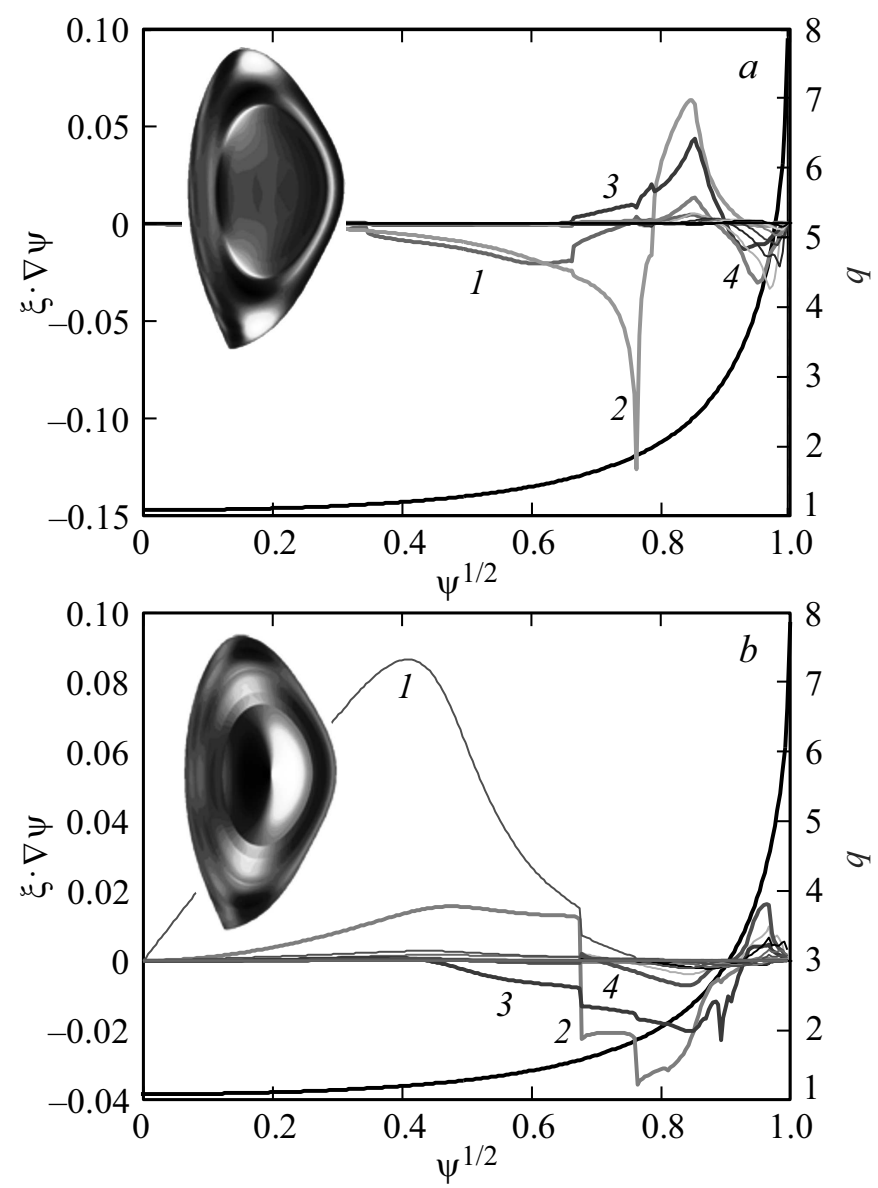

Рис. 3. Структура глобальных мод с низкими частотами в альфвеновском / звуковом спектре для сжимаемой плазмы: $a-89 \mathrm{kHz}, b-80 \mathrm{kHz}$. Цифры 1-4 - то же, что на рис. 2.

новского и звукового континуумов (рис. 1,b). Здесь кроме ветвей альфвеновского звукового континуума показаны также частоты альф-

Письма в ЖТФ, 2018, том 44, вып. 2 
веновской ветви в пределе медленного звука (сплошные линии) [5]. Код KINX был модифицирован для использования экспериментального профиля давления в слагаемом $\Gamma p|\nabla \cdot \xi|^{2}$ для сжимаемого смещения плазмы $\xi$ в интеграле возмущенной потенциальной энергии. Для сжимаемой плазмы с конечным давлением найдены аналоги глобальных мод с частотами, близкими к экспериментальным (показаны на рис. $1, b$ горизонтальными линиями). На рис. 3 показаны структуры таких мод с частотами и локализацией, соответствующими локальным максимумам ветвей альфвеновского звукового континуума. При этом мода с меньшей частотой и большой полоидальной гармоникой $m=1$ (рис. $3, b$ ), по-видимому, является результатом взаимодействия альфвеновских и звуковых мод, как и в случае BAAE-мод (beta-induced Alfven acoustic eigenmode) с более низкими частотами.

Результаты расчетов позволяют сделать вывод, что частоты колебаний, наблюдаемых в эксперименте, наиболее близки к частотам TAEмод, локализованных у границы плазмы в районе магнитных поверхностей с величинами фактора запаса устойчивости $q=2.5,3.5$. Частоты этих мод попадают в непрерывный спектр, что может приводить к их усиленному затуханию. Однако, как обсуждается в работе [6], коэффициенты затухания Ландау на тепловых ионах могут быть низкими из-за изменения поляризации моды при взаимодействии волны с энергичными частицами.

Работа выполнена при поддержке Российского научного фонда (грант № 17-12-01177).

\section{Список литературы}

[1] Петров Ю.В., Патров М.И., Гусев В.К., Иванов А.Е., Минаев В.Б., Сахаров Н.В., Толстяков С.Ю., Курскиев Г.С. // Физика плазмы. 2011. Т. 37. № 12. C. 1075-1080.

[2] Petrov Yu.V., Bakharev N.N., Gusev V.K., Minaev V.B., Kornev V.A., Kurskiev G.S., Patro M.I., Sakharov N.V., Tolstyakov S.Yu., Shchegolev P.B. // J. Plasma Phys. 2015. V. 81. P. 515810601.

[3] Петров Ю.В., Бахарев Н.Н., Гусев В.К., Минаев В.Б., Корнев В.А., Мельник А.Д., Патров М.И., Сахаров Н.В., Толстяков С.Ю., Курскиев Г.С., Чернышев Ф.В., Щеголев П.Б. // Письма в ЖТФ. 2014. Т. 40. В. 24. С. 99-106.

Письма в ЖТФ, 2018, том 44, вып. 2 
[4] Degtyarev L., Martynov A., Medvedev S., Troyon F., Villard L., Gruber R. // Comput. Phys. Commun. 1997. V. 103. P. 10-27.

[5] Cheng C.Z., Chance M.S. // Phys. Fluids. 1986. V. 11. P. 3695.

[6] Liu Y., Lin Z., Zhang H., Zhang W. // Nucl. Fusion. 2017. V. 57. P. 114001.

Письма в ЖТФ, 2018, том 44, вып. 2 